CNS CANCER

\title{
Tracing stem cells in oligodendroglioma
}

Intratumoural heterogeneity is a major cause of acquired anticancer drug resistance. The regulation of development and differentiation is a driver of tumour heterogeneity, as supported by evidence from studies of several malignancies (but mainly leukaemias). Among solid tumours, glioblastoma provides one of the best-established example, but limited information is available from other brain tumour types. A role for stem/

an opportunity for targeting

a specific cellular lineage progenitor cells in the pathogenesis of low-grade gliomas has now been demonstrated by the results of a recently published study.

To date, the main limitation for performing in vitro and in vivo studies of low-grade gliomas relates to the lack of suitable experimental models. For this study, Mario Suva and Aviv Regev relied on single-cell RNAsequencing (scRNA-seq) protocols because "from a single assay we can obtain information about cell type, cell state and genetic information expressed transcripts, which can then be used to reconstruct copy-number changes - all this at single-cell level and directly in samples from patients". Suva and Regev's teams have been the first to apply scRNA-seq for the analysis of cells derived from patients with oligodendroglioma. Three main expression patterns of cancer cells were consistently identified across six different tumours, evidencing the existence of oligodendrocytic and astrocytic differentiation programmes, as well as a stem/progenitor-cell population. Importantly, almost all the proliferating cells in the tumours could be assigned to the undifferentiated population.

These findings have important implications, as Suva explains: "oligodendrogliomas are slowgrowing tumours, but remain incurable. While we have a good understanding of the genetics of low-grade gliomas, our knowledge of the cell programmes driving their growth is limited." In their study, a cancer stem-cell population has been identified in oligodendrogliomas. Moreover, this population is the main source of proliferating cells in the tumour. In Suva's words, "our work provides evidence of the notion that these tumours are driven by the growth of a specific cell subpopulation. Our findings represent an opportunity for targeting a specific cellular lineage, for example, with immunotherapies. Differentiation therapies could also be beneficial for patients with oligodendroglioma."

Diana Romero

ORIGINAL ARTICLE Tirosh, I. et al. Single-cell RNA-seq supports a developmental hierarchy in human oligodendroglioma. Nature http://dx.doi.org/10.1038/nature20123 (2016) 